\title{
Long-term Follow-up Results of CT-guided Daily Adaptive Radiation Therapy for Localized Prostate Cancer
}

\author{
YUKIHIRO HAMA ${ }^{1,2}$ and TATSUMI KAJI ${ }^{2}$ \\ ${ }^{1}$ Department of Radiation Oncology, Tokyo-Edogawa Cancer Center, Edogawa Hospital, Tokyo, Japan; \\ ${ }^{2}$ Department of Radiology, National Defense Medical College, Saitama, Japan
}

\begin{abstract}
Background/Aim: To examine whether CT-guided daily adaptive radiation therapy (ART) can be safely administered against localized prostate cancer. Patients and Methods: Twenty-six patients with localized prostate adenocarcinoma were irradiated through opposed AP/PA fields up to $46 \mathrm{~Gy}$, then CT-guided daily ART was performed through opposed lateral fields until 76 Gy at 2 Gy/fraction. Results: Biochemical relapse-free survival was $89 \%$ at 3 years and $85 \%$ at 5 years and $76 \%$ at 7 years after ART, respectively. The 3-, 5- and 7-year local relapse-free survival rates were $100 \%, 100 \%$ and $95 \%$, respectively. The 5-year incidence rates of grade 1 and 2 late gastrointestinal adverse events were $15.4 \%(n=4)$ and $3.8 \%(n=1)$, respectively, and those of grade 1 and 2 late genitourinary adverse events were $3.8 \%(n=1)$ and $0 \%(n=0)$, respectively. Conclusion: $C T$-guided daily ART was well tolerated and suggested a good long-term tumor control with minimal adverse events.
\end{abstract}

Patients with prostate cancer often display large changes in the shape and size of their rectum during a course of radiotherapy, making adaptive radiotherapy (ART) appealing for this tumor site $(1,2)$. Since the anterior rectal wall tends to be the doselimiting structure when treating localized prostate cancer, administering sufficient dose to the prostate cancer while reducing excessive dose to the rectum is the key to successful treatment (3-6). Although it has been demonstrated that intensity-modulated radiotherapy (IMRT) is superior to nonimage-guided 3D conformal radiotherapy (CRT) (7), little consideration has been given to the feasibility of adaptive 3D

Correspondence to: Yukihiro Hama, MD, Ph.D., Department of Radiation Oncology, Tokyo-Edogawa Cancer Center, Edogawa Hospital, 2-24-18 Higashikoiwa, Edogawa-ku, Tokyo, 133-0052 Japan. Tel: +81 336731221, Fax: +81 336731229, e-mail: yjhama2005@yahoo.co.jp

Key Words: Precision medicine, prostatic neoplasms, radiation proctitis, adaptive radiotherapy.
CRT under image guidance. If radiation dose to the rectum can be lowered to an acceptable level while administering high dose radiation to planning target volume (PTV), 3D CRT is also considered to be a useful treatment option.

The purpose of this study was to investigate the feasibility of daily adaptive CT-guided 3D CRT for localized prostate cancer and present long-term follow-up results.

\section{Patients and Methods}

From February 2007 to November 2009, 26 patients with organconfined prostate cancer (c-T1c:2a:2b:2c:3a:3b=2:3:3:9:3:6, N0 M0) were prospectively enrolled and consented to an adaptive radiation treatment process in our institution. This study was conducted in accordance with the 1964 Helsinki Declaration, and the institutional review board granted a waiver of consent for this retrospective cohort study. The average age at diagnosis of prostate cancer was 71 years $(\mathrm{SD}=4.9$, range $=61-81)$. The average pretreatment prostate-specific antigen (PSA) level was $29.9 \mathrm{ng} / \mathrm{ml}$ (median=15.6, range $=5.3-157.0$ ). A total of $1,8,11$ and 6 prostate cancer patients were classified as low-, intermediate-, high- and very high-risk, respectively. Complications or coexisting disease that the registered patients originally had were arteriosclerosis (e.g., cerebral infarction, ischemic heart disease, arteriosclerosis obliterans) $(\mathrm{n}=8$, $31 \%)$, diabetes mellitus $(n=4,15 \%)$, fistula-in-ano $(n=1,4 \%)$, bladder cancer $(n=1,4 \%)$ and Parkinson's disease $(n=1,4 \%)$.

The initial PTV included the prostate and/or seminal vesicles, plus a $5-10 \mathrm{~mm}$ margin. Treatment is planned to deliver the prescribed dose to the PTV using a composition of two parallel opposed fields: AP (anterior posterior)/PA (posterior anterior) followed by lateral fields. Patients were irradiated through opposed AP/PA fields up to 46 Gy at $2 \mathrm{~Gy} /$ fraction, then CT-guided daily ART was performed through opposed lateral fields until 76 Gy at $2 \mathrm{~Gy} /$ fraction. All radiation treatments were performed with an imageguided radiation therapy (IGRT) system which combines a linear accelerator and a CT system on rails in the treatment room. When trying ART, in order to shorten the time to adjust the irradiation field, lead blocks of various angles were prepared in advance. Multileaf collimator was not used. The lead block was chosen so that the edge of the lead block was positioned on the boundary line between the prostate and the anterior rectal wall (Figure 1a and b).

During radiation therapy, patients met with a radiation oncologist once a week, after radiation treatment every 3 to 6 
a

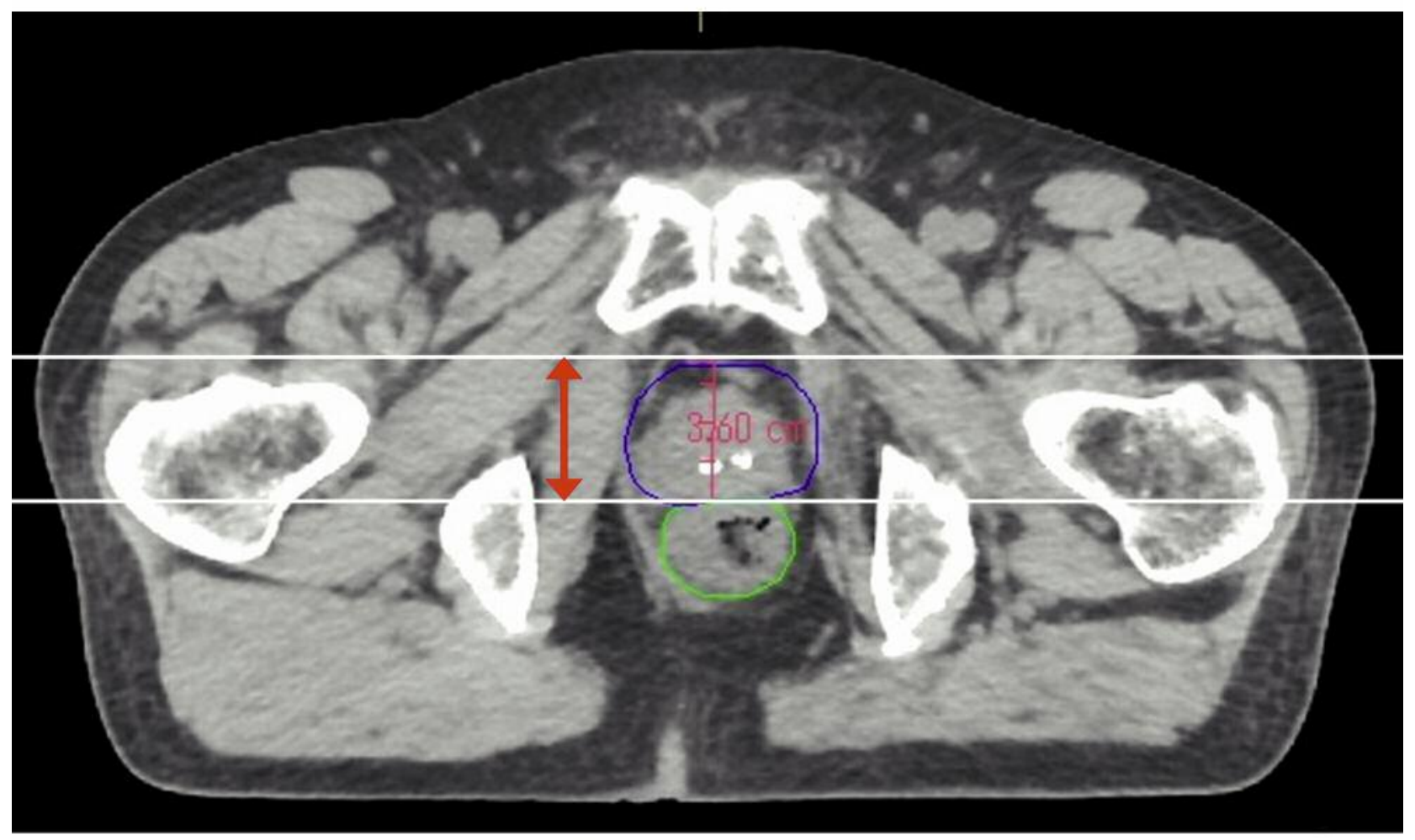

b



Figure 1. A CT scan was performed immediately before each ART using a diagnostic quality CT-on-rails. Then, the irradiation field size was determined in the anterior-posterior direction (a) and cranio-caudal direction (b) to include entire PTV (blue). Lead blocks of various angles (red, yellow) were chosen so that the edge of the lead block was positioned on the boundary line between the prostate and the anterior rectal wall (black arrows, rectum: green). 



Figure 2. Biochemical relapse-free survival (bRFS) (a), local relapse-free survival (LRFS) (b), disease-specific survival (DSS) (c) and overall survival $(\mathrm{OS})(d)$ estimates.

months. PSA was measured every visit and pelvic magnetic resonance imaging (MRI) was performed if necessary. Biochemical failure was defined according to the Phoenix criteria, a rise of 2 $\mathrm{ng} / \mathrm{ml}$ above nadir. Adverse events were assessed at every visit and were determined based on the NCI common terminology criteria for adverse events v2.0.

\section{Results}

Three intermediate-risk cases, 11 high-risk cases, and 6 very high-risk cases received neoadjuvant hormonal therapy (312 months, median 6 months). Five high-risk cases and 6 very high-risk cases received adjuvant hormonal therapy for 2 years or longer after ART. The median follow-up time was 104 months (range=38-115 months).
Biochemical relapse-free survival (bRFS) was $89 \%$ at 3 years and $85 \%$ at 5 years and $76 \%$ at 7 years after ART, respectively (Figure 2a). The 3-year, 5-year and 7-year local relapse-free survival rates were $100 \%, 100 \%$ and $95 \%$, respectively (Figure 2b). The 3-, 5- and 7-year disease-specific survival rates were $100 \%, 96 \%$ and $96 \%$, respectively (Figure $2 \mathrm{c})$. The 3-, 5- and 7-year overall survival rates were $100 \%$, $96 \%$ and $96 \%$, respectively (Figure $2 \mathrm{~d}$ ).

The 5-year incidence rates of grade 1 and 2 late gastrointestinal adverse events were $15.4 \%(n=4)$ and $3.8 \%$ $(n=1)$, respectively, and those of grade 1 and 2 late genitourinary adverse events were $3.8 \%(n=1)$ and $0 \%(n=0)$, respectively. One patient with grade 2 rectal toxicity had been originally treated with insulin-dependent diabetes mellitus 
(IDDM). Diabetes control was poor at any time before irradiation, during irradiation, after irradiation. Rectal bleeding was observed 16 months after irradiation was completed, and endoscopic examination showed radiation proctitis. When steroid ointment was administered to the rectum for 6 months, rectal bleeding ceased, and it was diagnosed as complete cure by the endoscope. Grade 3 or higher gastrointestinal or genitourinary adverse events were not observed.

\section{Discussion}

During a course of radiotherapy for localized prostate cancer, the size and shape of the rectum change daily (2). Even if IMRT is used, it cannot be adapted every time to the shape of the rectum that changes every day. If the irradiation field can be adjusted according to the shape of the rectum changing every day, it may be possible to sufficiently lower the rectal dose while maintaining the irradiation dose to PTV. So far, it has been shown that image-guided IMRT is more useful in reducing grade $\geq 2$ rectal toxicity than non-image-guided 3D CRT (7), but there is no clinical evidence comparing image-guided IMRT and image-guided 3D CRT. We speculated that if IGRT improves the accuracy of the radiation field placement, and ART adjusts the radiation fields according to the shape change of the rectum, rectal toxicity might be reduced to the similar extent as imageguided IMRT. The 5-year incidence of grade 2 rectal adverse events was $3.8 \%$ in this study, comparable to the incidence (5$20 \%)$ in previously reported IMRT studies $(8,9)$.

In order to accurately set up the radiation field by IGRT, it is essential to shorten the set-up time and increase the resolution of the image. CT-guided radiation therapy using a diagnostic CT scanner is a suitable method for high precision IGRT (10). In order to shorten the set-up time, the parallel opposed field is the most convenient method, and when carrying out the ART, it is possible to complete in a short time by preparing various types of shielding blocks in advance. The time required from CT imaging, patient set-up, selection of shields, to completion of irradiation is about 20 minutes. The treatment can be completed with a combination of two pairs (AP/PA, lateral) of parallel opposed beams and image guidance using pre-prepared shielding blocks. Therefore, complicated calculations like IMRT are not necessary.

The limitation of this study is that the number of patients was small $(n=26)$. However, given the fact that this study was conducted to examine the safety of new treatment method, the small number of patients is not a limitation. Secondary, patients with different relapse risks, different comorbidities have been analyzed together. Considering that the average age of treated patients is 71 years and that more than $30 \%$ of patients have diabetes or hypertension, it cannot be said that only patients who are unlikely to develop adverse events are selected.

In conclusion, this report showed the longest follow-up cohort of localized prostate cancer patients having received
CT-guided daily ART using parallel opposed fields. CT-guided daily ART was well tolerated and suggested good long-term tumor control with minimal adverse events, however, the hypothesis warrants further verification in future studies.

\section{Acknowledgements}

We would like to express our greatest thanks and appreciation to Dr. Katsumi Hayashi, Department of Radiation Oncology, the National Defense Medical College, Tokorozawa, Japan.

\section{References}

1 Thornqvist S, Hysing LB, Tuomikoski L, Vestergaard A, Tanderup K, Muren LP and Heijmen BJ: Adaptive radiotherapy strategies for pelvic tumors - a systematic review of clinical implementations. Acta Oncol 55(8): 943-958, 2016.

2 Wong JR, Grimm L, Uematsu M, Oren R, Cheng CW, Merrick S and Schiff P: Image-guided radiotherapy for prostate cancer by CTlinear accelerator combination: prostate movements and dosimetric considerations. Int J Radiat Oncol Biol Phys 61(2): 561-569, 2005.

3 Serrano NA, Kalman NS and Anscher MS Reducing rectal injury in men receiving prostate cancer radiation therapy: current perspectives. Cancer Manag Res 9: 339-350, 2017.

4 Buergy D, Sertdemir M, Weidner A, Shelan M, Lohr F, Wenz F, Schoenberg SO and Attenberger UI: Detection of local recurrence with 3-Tesla MRI after radical prostatectomy: A useful method for radiation treatment planning? In Vivo 32(1): 125-131, 2018.

5 Glaser SM, Kalash R, Bongiorni DR, Roberts MS, Balasubramani GK, Jacobs BL, Beriwal S, Heron DE and Greenberger JS: Challenges in the analysis of outcomes for surgical compared to radiotherapy treatment of prostate cancer. In Vivo 32(1): 113-120, 2018.

6 Pepe P, D’Urso D, Garufi A, Priolo G, Pennisi M, Russo G, Sabini MG, Valastro LM, Galia A and Fraggetta F: Multiparametric MRI apparent diffusion coefficient (ADC) accuracy in diagnosing clinically significant prostate cancer. In Vivo 31(3): 415-418, 2017.

7 Wortel RC, Incrocci L, Pos FJ, van der Heide UA, Lebesque JV, Aluwini S, Witte MG and Heemsbergen WD: Late side effects after image guided intensity modulated radiation therapy compared to 3D-conformal radiation therapy for prostate cancer: results from 2 prospective cohorts. Int J Radiat Oncol Biol Phys 95(2): 680-689, 2016.

8 Zelefsky MJ, Levin EJ, Hunt M, Yamada Y, Shippy AM, Jackson A and Amols HI: Incidence of late rectal and urinary toxicities after three-dimensional conformal radiotherapy and intensitymodulated radiotherapy for localized prostate cancer. Int J Radiat Oncol Biol Phys 70(4): 1124-1129, 2008.

9 Heemsbergen WD, Peeters ST, Koper PC, Hoogeman MS and Lebesque JV: Acute and late gastrointestinal toxicity after radiotherapy in prostate cancer patients: consequential late damage. Int J Radiat Oncol Biol Phys 66(1): 3-10, 2006.

10 Chen GP, Noid G, Tai A, Liu F, Lawton C, Erickson B and Li XL: Improving CT quality with optimized image parameters for radiation treatment planning and delivery guidance. Phys Imaging Radiat Oncol 4: 6-11, 2017.

Received September 11, 2018

Revised September 21, 2018

Accepted September 22, 2018 